\title{
Primary liver cancer
}

\author{
N Reau and FF Poordad (Editors) \\ Publisher: Humana Press; New York, Heidelberg, \\ Dordrecht, London; 295 pp. ISBN 978-1-61779-862-7; \\ $£ 135$
}

As several of the contributors point out in their introductory paragraphs, hepatocellular cancer (HCC) is the sixth most common cancer, and the third most common cause of cancer death worldwide. The majority of patients have co-existent liver disease and, in some patients, advanced cirrhosis may be the lifelimiting disease rather than cancer. Expert assessment of both the malignant and benign disease is therefore required in order to establish the optimal management, and a coordinated multidisciplinary approach is essential. Such an approach is reflected in the authorship of this book with contributions from surgeons, hepatologists, oncologists, radiologists and pathologists, although all are from North American institutions. The initial chapters deal with the aetiology of HCC and recommendations for surveillance. Unlike most cancers there are clear risk factors for the development of cirrhosis, which, once established, carries a 1-8\% risk per year of developing HCC. Furthermore, as early disease is potentially curable, HCC meets some of the key criteria for screening; namely, a defined at-risk population and effective therapy for early disease. The problem is that both the currently used modalities, ultrasound and $\alpha$-feto protein, have limited sensitivity and specificity for early disease. Chowdhury and Satoskar present an excellent overview of the evidence base for the current screening recommendations, and the chapters by Thomas and Oto on radiological diagnosis and by Pillai and Fimmel on emerging biomarkers inform the discussion. The HALT-C study, in which 1000 patients with Hepatitis C were followed prospectively for 4.3 years, demonstrated that the specificity and sensitivity of AFP at the time of diagnosis of HCC was 81 and $61 \%$ at $20 \mathrm{ng} \mathrm{ml}^{-1}$, while at $200 \mathrm{ng} \mathrm{ml}^{-1}$ the specificity increased to $100 \%$ at the expense of sensitivity, which decreased to $22 \%$. AFP is also affected by patient demographic and underlying liver disease. Alternative markers including the lens culinaris agglutinin glycoform (AFP-L3), des- $\gamma$-carboxy prothrombin, $\alpha$-L-fucosidase, glypican 3 and others remain unproven, and no blood-based biomarker is currently recommended by the AASLD, EASL or EORTC guidelines. Proteomic approaches and measurements of microRNAs have so far failed to yield reproducible and reliable alternatives. In terms of imaging, ultrasound has an overall specificity of $40-80 \%$ and sensitivity of $80-100 \%$, but only $25 \%$ sensitivity for early disease. Remarkably, only one randomised trial of screening has been performed and this was in China among an at-risk population of 18000 with hepatitis B. Despite less than $60 \%$ adhering to the protocol, there was a $37 \%$ reduction in HCC-related mortality. Taking the results of this trial, along with numerous non-randomised prospective studies, the current recommendations are for 6 monthly ultrasound, but there is clearly more to be done to improve both the accuracy of the screening test and the application to the at-risk population. Additionally, at $\$ 112993$ per life-year saved, the cost is prohibitive in developing countries with a high incidence. Another promising method to reduce the burden of disease is prevention of known risk factors, which is discussed in the chapter by Dharel and Lau. Worldwide, $52 \%$ of patients with HCC have a background of hepatitis B and only $40 \%$ have established cirrhosis. With the development of a vaccination to hepatitis B, primary prevention has proved valuable in endemic regions, resulting in a reduction of childhood HCC from 0.52 to $0.13 / 100000$ since its implementation in 1984. Unfortunately 350 million people remain infected, and secondary prevention using interferon- $\alpha$ has been shown in a randomised trial to reduce the risk of developing HCC from 13 to $2.7 \%$, although the benefit seems greater for those with suppressed HBV DNA. In the western countries and Japan, hepatitis $\mathrm{C}$ is present in $50 \%$ of those with HCC, but there is no established vaccine, and efforts have focussed on reducing transmission through the screening of blood products introduced in 1992. The risk from transfusion is now less than $0.03 \%$. In patients infected with hepatitis $C$, interferon- $\alpha$ that causes a sustained virological response, reduces the risk of HCC by $\sim 75 \%$. Other important risk factors, including cirrhosis secondary to alcohol and non-alcoholic fatty liver disease, are preventable with lifestyle modification. Despite the known and preventable risks, HCC-associated mortality has increased by 40\% between 1990 and 2004, while overall cancer deaths have declined by $18 \%$.

Pathological and radiological diagnosis is discussed, along with a useful chapter devoted to the differential diagnosis of a 'focal hepatic lesion'. The multitude of staging systems developed are also reviewed with the relative strengths and weaknesses discussed. The Barcelona Clinic Liver Cancer (BCLC) classification has proved to be one of the most widely used clinical staging system. It has been externally validated and is endorsed by both AASLD and EASL. In addition to prognostic information, the BCLC system provides a therapeutic algorithm that serves as a template for an overview of current therapies discussed by Susnow and colleagues. The accompanying chapter by Dhanasekaran and Cabrera examines emerging therapies that may demand modification of the BCLC system in the future. For early disease, the role of resection as a bridge to transplantation, expansion of the Milan criteria and the role of adjuvant therapy are considered. Novel ablative techniques are reviewed, although High Intensity Focussed Ultrasound (HIFU) is not mentioned. For intermediate disease, conventional transarterial chemoembolisation (TACE) 
using lipiodol/chemotherapy emulsion is increasingly being replaced with the use of drug-eluting beads, and the benefit of combining systemic therapy with TACE is being explored. Transarterial radioembolisation (TARE) seems to be comparable to TACE in non-randomised cohort comparisons, but is struggling to find a place in the BCLC algorithm in the absence of randomised trials. Perhaps the most active area of clinical investigation is in the management of advanced disease. The success of sorafenib has dispelled the prevailing therapeutic nihilism, and a number of pretenders have challenged sorafenib to the throne in recent years. Since this book was published, several of the mentioned trials have been presented and we now know that suntinib, brivanib and the combination of sorafenib and erlotinib have failed in the first-line randomised trials. Second-line treatment following failure or intolerance to sorafenib is an area of intense investigation with multiple new agents under investigation, although discussion of these agents and the rational for their use was somewhat brief.

The book also includes excellent reviews of paraneoplastic syndromes, liver cancer in children, liver anatomy and the management of recurrent disease. Molecular pathology was not included and, given the increasing emphasis on personalised medicine, it would have been interesting to review some of the recent data regarding molecular categorisation of HCC.

Overall, this book provides an excellent reference for students, trainees and members of a multidisciplinary team wishing to gain additional insights into the domain of their colleagues.

$\mathrm{T}_{\text {Meyer }}{ }^{1}$

${ }^{1}$ Senior Lecturer in Medical Oncology, UCL Cancer Institute, Paul O'Gorman Building, University College London, 72 Huntley Street, London WC1E 6BT, UK 